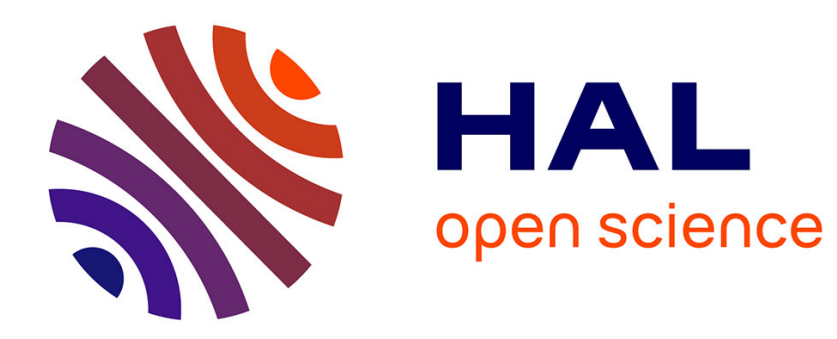

\title{
Homogenization of materials with sign changing coefficients
}

\author{
Renata Bunoiu, Karim Ramdani
}

\section{To cite this version:}

Renata Bunoiu, Karim Ramdani. Homogenization of materials with sign changing coefficients. Communications in Mathematical Sciences, 2016, 14 (4), pp.1137-1154. 10.4310/CMS.2016.v14.n4.a13 . hal-01162225v4

\section{HAL Id: hal-01162225 \\ https://hal.inria.fr/hal-01162225v4}

Submitted on 13 Apr 2016

HAL is a multi-disciplinary open access archive for the deposit and dissemination of scientific research documents, whether they are published or not. The documents may come from teaching and research institutions in France or abroad, or from public or private research centers.
L'archive ouverte pluridisciplinaire HAL, est destinée au dépôt et à la diffusion de documents scientifiques de niveau recherche, publiés ou non, émanant des établissements d'enseignement et de recherche français ou étrangers, des laboratoires publics ou privés. 


\title{
HOMOGENIZATION OF MATERIALS WITH SIGN CHANGING COEFFICIENTS*
}

\author{
RENATA BUNOIU ${ }^{\dagger}$ AND KARIM RAMDANI ${ }^{\ddagger}$
}

\begin{abstract}
We investigate a periodic homogenization problem involving two isotropic materials with conductivities of different signs: a classical material and a metamaterial (or negative material). Combining the T-coercivity approach and the unfolding method for homogenization, we prove wellposedness results for the initial and the homogenized problems and we obtain a convergence result. These results are obtained under the condition that the contrast between the two conductivities is large enough in modulus. The homogenized matrix, is generally anisotropic and indefinite, but it is shown to be isotropic and (positive or negative) definite for particular geometries having symmetries.
\end{abstract}

Key words. Metamaterials, homogenization, periodic unfolding, T-coercivity, indefinite operators.

AMS subject classifications. 35B27, 35Q60, 35M99, 78A48, 78M30.

\section{Introduction}

In optics, metamaterials (also known as negative or left-handed materials), have known a growing interest in the last two decades. These artificial composite materials exhibit the property of having negative dielectric permittivity and magnetic permeability in a certain range of frequency, leading hence to materials with negative refractive index and super lens effects. For a brief overview on metamaterials, we refer the interested reader to the review papers by Shamonina and Solymar [31] and Smith, Pendry, and Wiltshire [32]. From a mathematical viewpoint, Bouchitté et al. proposed a rigorous derivation and a mathematical justification of negative materials for Maxwell's system through a homogenization process (see $[9-11,19]$ ). Similar results have been established in phononics by Ávila et al. [4]. Motivated by the study of metamaterials, many authors have investigated the questions of well-posedness and numerical approximation of boundary value problems involving sign changing coefficients. For scalar transmission problems, let us mention the works of Bonnet-Ben Dhia et al. [5,7], Chesnel and Ciarlet Jr. [14], Chung and Ciarlet Jr. [15], Nicaise and Venel [25]. Time harmonic Maxwell's equations with sign changing coefficients have been investigated by Bonnet-Ben Dhia, Chesnel and Ciarlet Jr. [6], Fernandes and Raffetto [20], Oliveri and Raffetto [26]. For Maxwell's system in the time domain (see Li and Huang [23] and references therein).

More recently, physicists and mathematicians have been interested in periodic structures involving positive and negative materials, especially in the context of cloaking. For instance, Tricarico et al. [33] used alternating stacked plasmonic and non-plasmonic cylindrical layers to obtain Epsilon-Near-Zero (ENZ) metamaterials (i.e. metamaterials with close-to-zero values of the permittivity). Pendry and Ramakrishna [27] also used a stack of sign changing layers to refine the design of perfect lens. Jacob et al. [21] and Salandrino and Engheta [30] showed that a hyperlens can be designed using a multicoated cylinder (or sphere) with many sign changing thin coatings. A mathematical analysis of cloaking effects -when obtained via sign changing coefficients- can be found in Bouchitté and Schweizer [12], Milton and Nicorovici [24], Ammari et al. [3].

${ }^{*}$ Received: June 4, 2015; accepted (in revised form): September 26, 2015. Communicated by Olof Runborg.

${ }^{\dagger}$ Université de Lorraine and CNRS, Institut Élie Cartan de Lorraine, UMR 7502, Metz, F-57045, France (renata.bunoiu@univ-lorraine.fr).

${ }^{\ddagger}$ Inria, SPHINX, Villers-lès-Nancy, F-54600, France (karim.ramdani@inria.fr). 


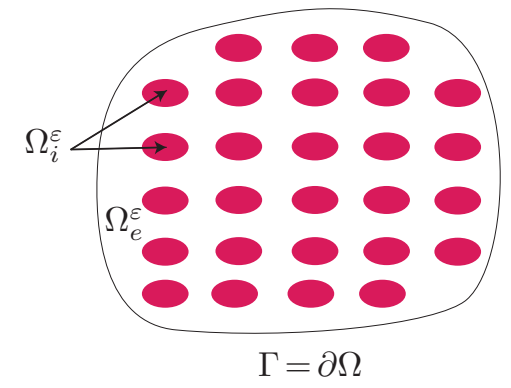

FIG. 1.1. The domain $\Omega=\overline{\Omega_{i}^{\varepsilon}} \cup \Omega_{e}^{\varepsilon}$ containing positive and negative materials.

In this work, we propose a mathematical analysis of the homogenization problem for indefinite systems, i.e. composite materials involving two materials with different signs. For the sake of simplicity, all the results are stated in dimension two, although they obviously hold true in higher dimension. Let us consider a bounded open domain $\Omega$ in $\mathbb{R}^{2}$ with Lipschitz boundary $\Gamma=\partial \Omega$. We assume that $\Omega$ contains $\varepsilon$-periodically distributed inclusions of size $\varepsilon>0$ occupying a domain denoted $\Omega_{i}^{\varepsilon}$. The domain exterior to the inclusions $\Omega_{e}^{\varepsilon}=\Omega \backslash \overline{\Omega_{i}^{\varepsilon}}$ is supposed to be connected (see Figure 1.1). Given $f \in H^{-1}(\Omega)$, consider the problem

$$
\left\{\begin{aligned}
-\operatorname{div}\left(\sigma^{\varepsilon} \nabla u^{\varepsilon}\right) & =f, & & \text { in } \Omega \\
u^{\varepsilon} & =0, & & \text { on } \Gamma .
\end{aligned}\right.
$$

where the matrix $\sigma^{\varepsilon}$ is given by

$$
\sigma^{\varepsilon}(x)=\left(\sigma_{e} \mathbb{1}_{\Omega_{e}^{\varepsilon}}(x)+\sigma_{i} \mathbb{1}_{\Omega_{i}^{\varepsilon}}(x)\right)\left(\begin{array}{ll}
1 & 0 \\
0 & 1
\end{array}\right),
$$

in which $\sigma_{e}$ and $\sigma_{i}$ are two real numbers satisfying

$$
\sigma_{i} \sigma_{e}<0 \text {. }
$$

This last condition represents the main difference with the classical elliptic case, whose homogenization is by now very well understood (for a short introduction, see for instance Allaire [2] and references therein). In the standard elliptic case, the well-posedness of the initial problem (1.1) and the homogenized problem (including the cell problems) are strongly based on the ellipticity of $\sigma^{\varepsilon}$. For sign changing matrices like the one considered in this paper, one has to obtain these well-posedness results in a different way. To achieve this, we use here the so-called $\mathbf{T}$-coercivity approach introduced in Bonnet, Ciarlet, and Zwölf [7] and we adapt it to the particular context of homogenization. We are then able to recover the same type of results known for the elliptic case provided the contrast between the two materials is large enough, i.e. when

$$
\kappa:=\left|\frac{\sigma_{e}}{\sigma_{i}}\right|>\kappa_{Y},
$$

for some constant $\kappa_{Y}$ depending only on the geometry of the reference cell $Y$. Note that the interior and exterior domains do not play symmetric roles due to the Dirichlet boundary condition on $\Gamma$. This is why we can not expect similar results for the case of low contrasts. 
The paper is organized as follows: Section 2 is devoted to the question of wellposedness. We prove that under condition (1.3), problem (1.1) admits for all $\varepsilon>0 \mathrm{a}$ unique solution $u^{\varepsilon} \in H_{0}^{1}(\Omega)$ which is uniformly bounded with respect to $\varepsilon$ (see Theorem 2.6). In Section 3, we derive the unfolded limit of problem (1.1) as $\varepsilon \rightarrow 0$ and we prove a convergence result (Theorem 3.1). The limit problem is proved to be well-posed (Theorem 3.2). Finally, Section 4 is devoted to the analysis of the homogenized problem.

\section{Some well-posedness results for sign changing problems}

This section is devoted to the proof of the well-posedness of the problem (1.1). Let us start with a more precise description of the geometry.

Without loss of generality, let $Y=[0,1]^{2}$ denote the reference cell and assume that $Y$ is composed of two materials: a dielectric material (positive material) and a metamaterial (negative material) located in the two subdomains $Y_{e}$ and $Y_{i}=Y \backslash \overline{Y_{e}}$ (see Figure 2.1). The positive material can be indifferently located in $Y_{e}$ or $Y_{i}$. We assume that the interface $\Sigma=\partial Y_{e} \cap \partial Y_{i}$ (which is also the boundary of $Y_{i}$ ) is Lipschitz and that $\Sigma \cap \partial Y=\varnothing$. Given $\sigma_{e}, \sigma_{i}$ such that $\sigma_{e} \sigma_{i}<0$, we define the $2 \times 2$ matrix-valued function $\sigma \in L^{\infty}\left(Y ; \mathbb{R}^{2 \times 2}\right)$ by

$$
\sigma(y)=\left(\sigma_{e} \mathbb{1}_{Y_{e}}(y)+\sigma_{i} \mathbb{1}_{Y_{i}}(y)\right)\left(\begin{array}{ll}
1 & 0 \\
0 & 1
\end{array}\right)
$$

For any $\varepsilon>0$ and any integer vector $k \in \mathbb{Z}^{2}$, we define the shifted cells:

$$
Y_{i}(\varepsilon, k)=\varepsilon\left(k+Y_{i}\right) .
$$

The interior periodic subdomain of $\Omega$ is then given by

$$
\Omega_{i}^{\varepsilon}=\bigcup_{k \in \mathbb{Z}}\left\{Y_{i}(\varepsilon, k) \mid Y_{i}(\varepsilon, k) \subset \Omega\right\}
$$

and we set

$$
\Omega_{e}^{\varepsilon}=\Omega \backslash \overline{\Omega_{i}^{\varepsilon}}
$$

With the above notation, the function $\sigma^{\varepsilon} \in L^{\infty}\left(\Omega ; \mathbb{R}^{2 \times 2}\right)$ defined by (1.2) can also be written as

$$
\sigma^{\varepsilon}(x)=\sigma\left(\frac{x}{\varepsilon}\right), \quad \forall x \in \Omega
$$

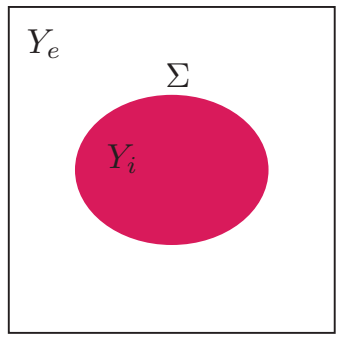

Fig. 2.1. The unit cell $Y$ composed of a positive and a negative material. 
2.1. Background on T-coercivity. The T-coercivity approach has been introduced in [7] to study the well-posedness of indefinite (i.e. sign changing) boundary value problems arising in electromagnetic wave propagation. We refer the interested reader to [5] for a more detailed discussion on the $\mathbf{T}$-coercivity and its use for the study of well-posedness of sign changing transmission problems. Approximation and convergence issues for sign changing problems can be found in [7]. Finally, let us emphasize that the T-coercivity has also been used in [8] to investigate the so-called interior transmission eigenvalue problem appearing in some scattering inverse problems [13].

Definition 2.1 (T-coercivity). Let $T \in \mathcal{L}(V)$ be an isomorphism on a Hilbert space $V$ equipped with the norm $\|\cdot\|$. A bilinear form $a(\cdot, \cdot)$ on $V \times V$ is called $\mathbf{T}$-coercive if there exists $\gamma>0$ such that

$$
|a(u, \mathbf{T} u)| \geqslant \gamma\|u\|^{2}
$$

The next theorem, which follows immediately from [7, Theorem 2.1], provides an abstract existence and uniqueness result for a family of variational problems depending on a parameter $\varepsilon$. The proof is given for reader's convenience.

THEOREM 2.1. Let $V$ be a Hilbert space equipped with the norm $\|\cdot\|$ and let $\left(\mathbf{T}^{\varepsilon}\right)_{\varepsilon>0}$ be a family of uniformly bounded linear invertible operators on $V$

$$
\left\|\mathbf{T}^{\varepsilon}\right\|_{\mathcal{L}(V)} \leqslant C, \quad \forall \varepsilon>0,
$$

for some constant $C>0$ independent of $\varepsilon$.

Let $\ell \in V^{\prime}$ and let $a^{\varepsilon}(\cdot, \cdot)$ be a bilinear form on $V$ such that there exists $\gamma, M>0$ such that

and

$$
\left|a^{\varepsilon}(u, v)\right| \leqslant M\|u\|\|v\|, \quad \forall u, v \in V
$$

$$
\left|a^{\varepsilon}\left(u, \mathbf{T}^{\varepsilon} u\right)\right| \geqslant \gamma\|u\|^{2}, \quad \forall u \in V .
$$

Then, the variational problem

$$
\text { Find } u^{\varepsilon} \in V \text { such that }: a^{\varepsilon}\left(u^{\varepsilon}, v\right)=\ell(v), \quad \forall v \in V
$$

admits for all $\varepsilon>0$ a unique solution $u^{\varepsilon} \in V$ and there exists $C^{*}>0$ independent of $\varepsilon$ such that

$$
\left\|u^{\varepsilon}\right\| \leqslant C^{*}\|\ell\|_{V^{\prime}}
$$

Proof. Since $\mathbf{T}^{\varepsilon}$ is invertible, problem (2.2) is equivalent to the variational problem

$$
\text { Find } u^{\varepsilon} \in V \text { such that }: a^{\varepsilon}\left(u^{\varepsilon}, \mathbf{T}^{\varepsilon} v\right)=\ell\left(\mathbf{T}^{\varepsilon} v\right), \quad \forall v \in V \text {. }
$$

By assumption, the bilinear form $b^{\varepsilon}(\cdot, \cdot):=a^{\varepsilon}\left(\cdot, \mathbf{T}^{\varepsilon} \cdot\right)$ satisfies then for all $u, v \in V$ :

$$
\left|b^{\varepsilon}(u, v)\right| \leqslant M C\|u\|\|v\| \quad \quad\left|b^{\varepsilon}(u, u)\right| \geqslant \gamma\|u\|^{2} .
$$

Lax-Milgram lemma applied to the coercive bilinear form $b^{\varepsilon}(\cdot, \cdot):=a^{\varepsilon}\left(\cdot, \mathbf{T}^{\varepsilon} \cdot\right)$ and the linear form $\ell^{\varepsilon}(\cdot):=\ell\left(\mathbf{T}^{\varepsilon} \cdot\right)$ shows the existence of a unique $u^{\varepsilon} \in V$ satisfying (2.3). Moreover, setting $C^{*}=C / \gamma$, we have 


$$
\left\|u^{\varepsilon}\right\| \leqslant \frac{1}{\gamma}\left\|\ell^{\varepsilon}\right\|_{V^{\prime}} \leqslant C^{*}\|\ell\|_{V^{\prime}}
$$

In order to apply this abstract result to our problem (1.1), the main difficulty is the construction of a family of operators $\left(\mathbf{T}^{\varepsilon}\right)_{\varepsilon>0}$ such that the uniform $\mathbf{T}$-coercivity condition (2.1) holds true. To do so, we need two preliminary technical results on extension operators in the reference cell $Y$ (from $Y_{e}$ to $Y$ ) and in $\Omega$ (from $\Omega_{e}^{\varepsilon}$ to $\Omega$ ). This is done in the next subsection.

\subsection{Extension operators.}

Lemma 2.2. Let $P$ denote the harmonic extension operator from $Y_{e}$ to $Y$. That is, for all $u \in H^{1}\left(Y_{e}\right),(P u)_{\mid Y_{e}}=u$ in $Y_{e}$ and $(P u)_{\mid Y_{i}}$ satisfies:

$$
\left\{\begin{aligned}
-\Delta(P u)=0 & \left(Y_{i}\right) \\
P u & =u
\end{aligned}\right.
$$

Then, the operator $P \in \mathcal{L}\left(H^{1}\left(Y_{e}\right) ; H^{1}(Y)\right)$ satisfies the following conditions

1. Affine functions are invariant by $P$ : for all $\xi \in \mathbb{R}^{2}$ and all $\gamma \in \mathbb{R}$, we have

$$
\left(P \varphi_{\xi, \gamma}\right)(y)=\varphi_{\xi, \gamma}(y), \quad \forall y \in Y,
$$

where $\varphi_{\xi, \gamma}(y)=\xi \cdot y+\gamma$.

2. There exists $\kappa_{Y}>0$ depending only on the geometry of $Y_{e}$ such that for all $u \in H^{1}\left(Y_{e}\right)$ :

$$
\|\nabla(P u)\|_{L^{2}\left(Y_{i}\right)}^{2} \leqslant \kappa_{Y}\|\nabla u\|_{L^{2}\left(Y_{e}\right)}^{2} .
$$

Proof. The first assertion simply follows from the fact that affine functions are harmonic. To prove the second one, we note that

$$
P u=P\left(u-\mathcal{M}_{Y_{e}}(u)\right)+\mathcal{M}_{Y_{e}}(u),
$$

where the constant $\mathcal{M}_{Y_{e}}(u)=\left|Y_{e}\right|^{-1} \int_{Y_{e}} u$ denotes the mean value of $u$ on $Y_{e}$. Consequently, we have

$$
\begin{aligned}
\|\nabla(P u)\|_{L^{2}\left(Y_{i}\right)} & =\left\|\nabla\left(P\left(u-\mathcal{M}_{Y_{e}}(u)\right)\right)\right\|_{L^{2}\left(Y_{i}\right)} \\
& \leqslant\left\|P\left(u-\mathcal{M}_{Y_{e}}(u)\right)\right\|_{H^{1}(Y)} \\
& \leqslant\|P\|_{\mathcal{L}\left(H^{1}\left(Y_{e}\right) ; H^{1}(Y)\right)}\left\|u-\mathcal{M}_{Y_{e}}(u)\right\|_{H^{1}\left(Y_{e}\right)} \\
& \leqslant \sqrt{1+C_{\mathrm{PW}}\left(Y_{e}\right)^{2}}\|P\|_{\mathcal{L}\left(H^{1}\left(Y_{e}\right) ; H^{1}(Y)\right)}\left\|\nabla\left(u-\mathcal{M}_{Y_{e}}(u)\right)\right\|_{L^{2}\left(Y_{e}\right)} \\
& =\sqrt{1+C_{\mathrm{PW}}\left(Y_{e}\right)^{2}}\|P\|_{\mathcal{L}\left(H^{1}\left(Y_{e}\right) ; H^{1}(Y)\right)}\|\nabla u\|_{L^{2}\left(Y_{e}\right)},
\end{aligned}
$$

where we have used the Poincaré-Wirtinger inequality in $Y_{e}$

This shows (2.5) with

$$
\left\|u-\mathcal{M}_{Y_{e}}(u)\right\|_{L^{2}\left(Y_{e}\right)} \leqslant C_{\mathrm{PW}}\left(Y_{e}\right)\|\nabla u\|_{L^{2}\left(Y_{e}\right)}, \quad \forall u \in H^{1}\left(Y_{e}\right) .
$$

$$
\kappa_{Y}=\left(1+C_{\mathrm{PW}}\left(Y_{e}\right)^{2}\right)\|P\|_{\mathcal{L}\left(H^{1}\left(Y_{e}\right) ; H^{1}(Y)\right)}^{2}
$$

REMARK 2.3. Among all the possible extension operators from $Y_{e}$ to $Y$ satisfying an estimate of the form (2.5), one may wonder which operator leads to the smallest 
constant $\kappa_{Y}$. It turns out that it is the harmonic extension operator $P$ defined by (2.4). Indeed, it follows immediately from (2.4) that

$$
\int_{Y_{i}} \nabla(P u) \cdot \nabla v=0, \quad \forall v \in H_{0}^{1}\left(Y_{i}\right) .
$$

Therefore, for any extension operator $R \in \mathcal{L}\left(H^{1}\left(Y_{e}\right) ; H^{1}(Y)\right)$ (i.e. such that $\left.(R u)_{\mid Y_{e}}=u\right)$ :

$$
\int_{Y_{i}}|\nabla(R u)|^{2}=\int_{Y_{i}}|\nabla(P u)-\nabla(P u-R u)|^{2}=\int_{Y_{i}}|\nabla(P u)|^{2}+\int_{Y_{i}}|\nabla(P u-R u)|^{2},
$$

since $(P u-R u)_{\mid Y_{i}} \in H_{0}^{1}\left(Y_{i}\right)$. Consequently, we have

$$
\|\nabla(P u)\|_{L^{2}\left(Y_{i}\right)} \leqslant\|\nabla(R u)\|_{L^{2}\left(Y_{i}\right)}, \quad \forall u \in H^{1}\left(Y_{e}\right) .
$$

Using this harmonic extension operator $P$ for the reference cell, we are able to construct a family of uniformly bounded extension operators from $\Omega_{e}^{\varepsilon}$ to $\Omega$. To do this, we use a classical result from homogenization theory (see e.g. [18, Theorem 2.10]), which is recalled below, whose proof is given to make explicit the dependence of the constant appearing in the estimate (2.6). In particular, it turns out that the (uniform) bound for the family of extension operators from $\Omega_{e}^{\varepsilon}$ to $\Omega$ is the same as the one in the reference cell (and this fact is important to obtain the well-posedness results of subsection 2.4).

Proposition 2.4. Let

$$
H_{0, \Gamma}^{1}\left(\Omega_{e}^{\varepsilon}\right)=\left\{u \in H^{1}\left(\Omega_{e}^{\varepsilon}\right) \mid u_{\mid \Gamma}=0\right\} .
$$

Then, there exists a family of continuous extension operators $P^{\varepsilon} \in \mathcal{L}\left(H_{0, \Gamma}^{1}\left(\Omega_{e}^{\varepsilon}\right) ; H_{0}^{1}(\Omega)\right)$ such that

$$
\left(P^{\varepsilon} u\right)(x)=u(x), \quad \forall x \in \Omega^{\varepsilon}
$$

and

$$
\left\|\nabla\left(P^{\varepsilon} u\right)\right\|_{L^{2}(\Omega)}^{2} \leqslant \kappa_{Y}\|\nabla u\|_{L^{2}\left(\Omega_{e}^{\varepsilon}\right)}^{2}
$$

where $\kappa_{Y}$ is the constant given by Lemma 2.2.

Proof. Given $u \in H_{0, \Gamma}^{1}\left(\Omega_{e}^{\varepsilon}\right)$, we define for all $k \in \mathbb{Z}^{2}$ the function $u_{k}^{\varepsilon} \in H^{1}\left(Y_{e}\right)$ by setting

$$
u_{k}^{\varepsilon}(y)=u(\varepsilon(k+y)), \quad \forall y \in Y_{e}
$$

Set

$$
\left(P^{\varepsilon} u\right)(x):=\left(P u_{k}^{\varepsilon}\right)\left(\frac{x}{\varepsilon}-k\right), \quad \forall x \in \Omega_{e}^{\varepsilon},
$$

where $P$ denotes the harmonic extension operator defined in Lemma 2.2. This operator clearly defines a bounded map from $H_{0, \Gamma}^{1}\left(\Omega_{e}^{\varepsilon}\right)$ to $H_{0}^{1}(\Omega)$. Moreover, on each cell $\varepsilon\left(k+Y_{i}\right)$, $k \in \mathbb{Z}^{2}$, we have

$$
\begin{aligned}
\int_{\varepsilon\left(k+Y_{i}\right)}\left|\nabla\left(P^{\varepsilon} u\right)(x)\right|^{2} \mathrm{~d} x & =\int_{Y_{i}}\left|\varepsilon^{-1} \nabla\left(P u_{k}^{\varepsilon}\right)(y)\right|^{2} \varepsilon^{2} \mathrm{~d} y \\
& \leqslant \kappa_{Y} \int_{Y_{e}}\left|\varepsilon^{-1} \nabla u_{k}^{\varepsilon}(y)\right|^{2} \varepsilon^{2} \mathrm{~d} y \\
& =\int_{\varepsilon\left(k+Y_{i}\right)}|\nabla u(x)|^{2} \mathrm{~d} x
\end{aligned}
$$

The result follows by summing the above inequalities over $k$. 
2.3. Well-posedness in the reference cell. Before establishing the wellposedness of problem (1.1), we first state a well-posedness result for a similar problem set in the reference cell with periodic boundary conditions. Solving this simpler problem (which appears naturally for the study of the so-called cell problems, see Section 4) allows us to highlight the main steps of the proof for problem(1.1).

Let

$$
W^{\#}:=\left\{u \in H_{\#}^{1}(Y) ; \int_{Y} u=0\right\} \subset H^{1}(Y)
$$

where $H_{\#}^{1}(Y)$ is the subspace of $H^{1}(Y)$ constituted of functions satisfying periodic boundary conditions on $\partial Y$. Thanks to Poincaré-Wirtinger inequality, the space $W^{\#}$ defines a Hilbert space when endowed with the norm

$$
\|u\|_{\#}:=\|\nabla u\|_{L^{2}(Y)} .
$$

For $f \in\left(H^{1}(Y)\right)^{\prime}$, we investigate the well-posedness of the problem

$$
\text { Find } u \in W^{\#} \text { such that: } \quad-\operatorname{div}(\sigma \nabla u)=f \text {. }
$$

Setting

$$
a(u, v):=\int_{Y} \sigma(y) \nabla u(y) \cdot \nabla v(y) \mathrm{d} y, \quad \forall u, v \in H^{1}(Y),
$$

the weak formulation of problem (2.8) reads

$$
\text { Find } u \in W^{\#} \text { such that: } \quad a(u, v)=\langle f, v\rangle, \quad \forall v \in W^{\#} \text {. }
$$

Throughout the paper, duality will be denoted by integrals for the sake of simplicity.

THEOREM 2.5. Let $\kappa_{Y}$ denote the constant in (2.6). If the contrast $\kappa:=\left|\frac{\sigma_{e}}{\sigma_{i}}\right|$ satisfies

$$
\kappa>\kappa_{Y}
$$

then boundary value problem (2.8) (or equivalently its variational formulation (2.10)) admits a unique solution $u \in W^{\#}$ depending continuously on $f$ for all $f \in\left(H^{1}(Y)\right)^{\prime}$ satisfying the compatibility condition $\langle f, 1\rangle=0$.

Proof. Without loss of generality, we may assume that $\sigma_{e}>0$ and $\sigma_{i}<0$ so that $\kappa=\sigma_{e} /\left|\sigma_{i}\right|$. Let $\mathbf{T}_{Y}$ be the operator defined on $H^{1}(Y)$ by

$$
\mathbf{T}_{Y} u= \begin{cases}u_{\mid Y_{e}} & \text { in } Y_{e} \\ -u_{\mid Y_{i}}+2 P\left(u_{\mid Y_{e}}\right) & \text { in } Y_{i} .\end{cases}
$$

We clearly have $\mathbf{T}_{Y} \in \mathcal{L}\left(W^{\#}\right)$ and $\left(\mathbf{T}_{Y}\right)^{2}=\mathrm{Id}$. Moreover, for all $u \in W^{\#}$ and for all $\eta>0$, we have

$$
\begin{aligned}
a\left(u, \mathbf{T}_{Y} u\right) & =\sigma_{e} \int_{Y_{e}}|\nabla u(y)|^{2} \mathrm{~d} y+\left|\sigma_{i}\right| \int_{Y_{i}}|\nabla u(y)|^{2} \mathrm{~d} y+2 \sigma_{i} \int_{Y_{i}} \nabla u(y) \cdot \nabla(P u)(y) \mathrm{d} y \\
& \geqslant \sigma_{e}\|\nabla u\|_{L^{2}\left(Y_{e}\right)}^{2}+\left|\sigma_{i}\right|\|\nabla u\|_{L^{2}\left(Y_{i}\right)}^{2}-\left|\sigma_{i}\right| \eta\|\nabla u\|_{L^{2}\left(Y_{i}\right)}^{2}-\frac{\left|\sigma_{i}\right|}{\eta}\|\nabla(P u)\|_{L^{2}\left(Y_{i}\right)}^{2}<
\end{aligned}
$$


where we have used Young's inequality for the last estimate. Consequently, using (2.5), we obtain that

$$
a\left(u, \mathbf{T}_{Y} u\right) \geqslant\left|\sigma_{i}\right|\left\{\left(\kappa-\frac{\kappa_{Y}}{\eta}\right)\|\nabla u\|_{L^{2}\left(Y_{e}\right)}^{2}+(1-\eta)\|\nabla u\|_{L^{2}\left(Y_{i}\right)}^{2}\right\} .
$$

Thus, if the contrast satisfies $\kappa>\kappa_{Y}$, we can choose $\eta \in\left(\kappa_{Y} / \kappa, 1\right)$ and get the existence of a constant $\gamma>0$ such that

$$
a\left(u, \mathbf{T}_{Y} u\right) \geqslant \gamma\|\nabla u\|_{L^{2}(Y)}^{2}=\gamma\|u\|_{\#}^{2}
$$

for all $W^{\#}$. The conclusion follows then immediately by applying Theorem 2.1 (or more precisely its simpler version corresponding to the case where the bilinear form is independent of $\varepsilon$ ).

2.4. Well-posedness of the problem in $\Omega$. For $f \in H^{-1}(\Omega)$, we investigate the well-posedness in $H_{0}^{1}(\Omega)$ of the problem

$$
-\operatorname{div}\left(\sigma^{\varepsilon} \nabla u^{\varepsilon}\right)=f .
$$

The weak formulation of the above problem reads as follows:

$$
\begin{gathered}
\text { Find } u^{\varepsilon} \in H_{0}^{1}(\Omega) \text { such that } \\
a^{\varepsilon}\left(u^{\varepsilon}, v\right)=\langle f, v\rangle_{H^{-1}(\Omega), H_{0}^{1}(\Omega)} \quad \forall v \in H_{0}^{1}(Y),
\end{gathered}
$$

where the bilinear form $a^{\varepsilon}(\cdot, \cdot)$ is defined on $H_{0}^{1}(\Omega)$ by

$$
a^{\varepsilon}(u, v):=\int_{\Omega} \sigma^{\varepsilon}(x) \nabla u(x) \cdot \nabla v(x) \mathrm{d} x, \quad \forall u, v \in H_{0}^{1}(\Omega) .
$$

Using the family of extension operators $\left(P^{\varepsilon}\right)$ obtained in Proposition 2.4, we are able now to construct a family of uniformly bounded operators $\left(\mathbf{T}^{\varepsilon}\right)$ and to apply Theorem 2.1. This leads to the main result of this section.

THEOREM 2.6. Let $\kappa_{Y}$ denote the constant in (2.6). If the contrast $\kappa:=\left|\frac{\sigma_{e}}{\sigma_{i}}\right|$ satisfies

$$
\kappa>\kappa_{Y}
$$

then equation (2.12) admits a unique solution $u^{\varepsilon} \in H_{0}^{1}(\Omega)$ for all $f \in H^{-1}(\Omega)$. Moreover, there exists a constant $C>0$ such that

$$
\left\|\nabla u^{\varepsilon}\right\|_{L^{2}(\Omega)} \leqslant C\|f\|_{H^{-1}(\Omega)},
$$

for all $\varepsilon>0$.

Proof. Since the proof is quite similar to the proof of Theorem 2.5, we only sketch the main differences. Assuming once again for simplicity that $\sigma_{e}>0$ and $\sigma_{i}<0$ (so that $\left.\kappa=\sigma_{e} /\left|\sigma_{i}\right|\right)$, let us define for all $\varepsilon>0$ the following operator on $H_{0}^{1}(\Omega)$ (endowed with the norm $\left.\|\nabla \cdot\|_{L^{2}(\Omega)}\right)$ :

$$
\left(\mathbf{T}^{\varepsilon} u\right)(x)= \begin{cases}u(x) & \text { for } x \in \Omega_{e}^{\varepsilon} \\ -u(x)+2\left(P^{\varepsilon} u\right)(x) & \text { for } x \in \Omega_{i}^{\varepsilon}\end{cases}
$$


We clearly have $\mathbf{T}^{\varepsilon} \in \mathcal{L}\left(H_{0}^{1}(\Omega)\right),\left(\mathbf{T}^{\varepsilon}\right)^{2}=$ Id and the family of operators $\left(\mathbf{T}^{\varepsilon}\right)_{\varepsilon>0}$ is uniformly bounded in $\mathcal{L}\left(H_{0}^{1}(\Omega)\right.$ ) due to the estimate (2.6) of Proposition 2.4. Moreover, for all $u \in H_{0}^{1}(\Omega)$ and all $\eta>0$, we have

$$
a^{\varepsilon}\left(u, \mathbf{T}^{\varepsilon} u\right) \geqslant \sigma_{e}\|\nabla u\|_{L^{2}\left(\Omega_{e}^{\varepsilon}\right)}^{2}+\left|\sigma_{i}\right|\|\nabla u\|_{L^{2}\left(\Omega_{i}^{\varepsilon}\right)}^{2}-\left|\sigma_{i}\right| \eta\|\nabla u\|_{L^{2}\left(\Omega_{i}^{\varepsilon}\right)}^{2}-\frac{\left|\sigma_{i}\right|}{\eta}\left\|\nabla\left(P^{\varepsilon} u\right)\right\|_{L^{2}\left(\Omega_{i}^{\varepsilon}\right)}^{2} .
$$

Using (2.6), this yields

$$
a^{\varepsilon}\left(u, \mathbf{T}^{\varepsilon} u\right) \geqslant\left|\sigma_{i}\right|\left\{\left(\kappa-\frac{\kappa_{Y}}{\eta}\right)\|\nabla u\|_{L^{2}\left(\Omega_{e}^{\varepsilon}\right)}^{2}+(1-\eta)\|\nabla u\|_{L^{2}\left(\Omega_{i}^{\varepsilon}\right)}^{2}\right\} .
$$

Hence, for $\kappa>\kappa_{Y}$, there exists a constant $\gamma>0$ such that for all $u \in H_{0}^{1}(\Omega)$ and all $\varepsilon>0$ :

$$
a^{\varepsilon}\left(u, \mathbf{T}^{\varepsilon} u\right) \geqslant \gamma\|\nabla u\|_{L^{2}(\Omega)}^{2}
$$

The conclusion follows then from Theorem 2.1.

\section{Convergence analysis}

In this section, using the uniform estimate (2.15), we first pass to the limit in problem (1.1) (more precisely in its weak form (2.13)) using the unfolding method for periodic homogenization. For this step, the non-ellipticity of $\sigma^{\varepsilon}$ plays no role: the proof is the same as in the elliptic case. However, we prefer to include it for the reader's convenience. The obtained limit problem involves two scales (a macroscopic one and a microscopic one) and is indefinite. It is proved (see Theorem 3.2) to be well-posed for high contrasts using the $\mathbf{T}$-coercivity.

3.1. Derivation of the unfolded limit problem. In order to pass to the limit as $\varepsilon$ tends to zero in problem (2.13), we will use the unfolding method, introduced in [16]. The idea of the unfolding method is to transform oscillating functions defined on the domain $\Omega$ into functions defined on the domain $\Omega \times Y$, in order to isolate the oscillations in the second variable. This transformation, together with a priori estimates, will allow us to use compactness results and then to get the limits of $u_{\varepsilon}$ when $\varepsilon$ tends to zero. We first recall some results concerning the unfolding operator that we use in the sequel.

For every real number $a$, we introduce the decomposition $a=[a]+\{a\}$ where $[a]$ denotes the integer part of $a$ and $\{a\} \in[0,1)$ its fractional part. By analogy, we can write every $x=\left(x_{1}, x_{2}\right) \in \mathbb{R}^{2}$ using the decomposition of $x_{i} / \varepsilon, i=1,2$ which yields

$$
x=\varepsilon\left[\frac{x}{\varepsilon}\right]_{Y}+\varepsilon\left\{\frac{x}{\varepsilon}\right\}_{Y}, \quad\left[\frac{x}{\varepsilon}\right]_{Y} \in \mathbb{Z}^{2},\left\{\frac{x}{\varepsilon}\right\}_{Y} \in Y .
$$

Definition 3.1. For every $w \in L^{2}(\Omega)$, extended by zero outside $\Omega$, the unfolding operator $\mathcal{T}_{\varepsilon}$ is defined by

$$
\mathcal{T}_{\varepsilon}(w)(x, y)=w\left(\varepsilon\left[\frac{x}{\varepsilon}\right]_{Y}+\varepsilon y\right), \quad x \in \Omega, y \in Y .
$$

According to [16], this operator has the following properties:

$\left(\mathbf{P}_{1}\right) \mathcal{T}_{\varepsilon}$ is linear and continuous from $L^{2}(\Omega)$ to $L^{2}(\Omega \times Y)$;

$\left(\mathbf{P}_{2}\right) \mathcal{T}_{\varepsilon}(\varphi \psi)=\mathcal{T}_{\varepsilon}(\varphi) T_{\varepsilon}(\psi), \forall \varphi, \psi \in L^{2}(\Omega) ;$

$\left(\mathbf{P}_{3}\right)$ If $\varphi_{\varepsilon} \in L^{2}(\Omega)$ and $\varphi_{\varepsilon} \rightarrow \varphi$ strongly in $L^{2}(\Omega)$, then

$$
\mathcal{T}_{\varepsilon}\left(\varphi_{\varepsilon}\right) \rightarrow \varphi \text { strongly in } L^{2}(\Omega \times Y) ;
$$


$\left(\mathbf{P}_{4}\right)$ If $\varphi \in L^{2}(Y)$ is a $Y$-periodic function and $\varphi^{\varepsilon}(x)=\varphi\left(\frac{x}{\varepsilon}\right)$ then

$$
\mathcal{T}_{\varepsilon}\left(\varphi_{\left.\right|_{\Omega}}^{\varepsilon}\right) \rightarrow \varphi \text { strongly in } L^{2}(\Omega \times Y)
$$

In what follows, in order to replace integrals over the domain $\Omega$ by integrals over the domain $\Omega \times Y$, we use the following classical relation

$$
\int_{\Omega} \varphi \mathrm{d} x \sim \frac{1}{|Y|} \int_{\Omega \times Y} \mathcal{T}_{\varepsilon}(\varphi) \mathrm{d} x \mathrm{~d} y, \quad \forall \varphi \in L^{1}(\Omega),
$$

where the symbol $\sim$ means that the difference between the two integrals tends to 0 as $\varepsilon$ goes to 0 (throughout, we prefer to keep the term $|Y|$ in the formulae for the sake of generality, although we obviously have for our particular choice of unit cell $|Y|=1$ ). Indeed, for every cell $\varepsilon \xi+\varepsilon Y, \xi \in \mathbb{Z}^{2}$ strictly included in $\Omega$, we have

$$
\int_{\varepsilon \xi+\varepsilon Y} \varphi(x) \mathrm{d} x=\varepsilon^{2} \int_{Y} \varphi(\varepsilon \xi+\varepsilon y) \mathrm{d} y=\frac{1}{|Y|} \int_{(\varepsilon \xi+\varepsilon Y) \times Y} \mathcal{T}_{\varepsilon}(\varphi)(x, y) \mathrm{d} x \mathrm{~d} y .
$$

By using this equality in every cell strictly included in $\Omega$ and by denoting as $\widehat{\Omega}_{\varepsilon}$ the largest union of such $\varepsilon \xi+\varepsilon Y$ cells strictly included in $\Omega$, the following exact formula is obtained

$$
\int_{\widehat{\Omega}_{\varepsilon}} \varphi(x) \mathrm{d} x=\frac{1}{|Y|} \int_{\widehat{\Omega}_{\varepsilon} \times Y} \mathcal{T}_{\varepsilon}(\varphi)(x, y) \mathrm{d} x \mathrm{~d} y
$$

yielding

$$
\left|\int_{\Omega} \varphi(x) \mathrm{d} x-\frac{1}{|Y|} \int_{\Omega \times Y} \mathcal{T}_{\varepsilon}(\varphi)(x, y) \mathrm{d} x \mathrm{~d} y\right| \leq 2\|\varphi\|_{L^{1}\left(\Omega \backslash \widehat{\Omega}_{\varepsilon}\right)},
$$

which shows (3.1).

We can now state the main result of this section.

THEOREM 3.1. Let $f \in H^{-1}(\Omega)$ be given and let $u^{\varepsilon}$ be the solution of problem (2.13). Then there exist $u_{0} \in H_{0}^{1}(\Omega)$ and $u_{1} \in L^{2}\left(\Omega ; H_{\#}^{1}(Y)\right)$ with $\mathcal{M}_{Y}\left(u_{1}\right)=0$ such that, up to a subsequence, the following assertions hold true:

1. $u^{\varepsilon} \rightarrow u_{0} \quad$ weakly in $H_{0}^{1}(\Omega)$.

2. $\mathcal{T}_{\varepsilon}\left(u^{\varepsilon}\right) \rightarrow u_{0} \quad$ weakly in $L^{2}\left(\Omega, H^{1}(Y)\right)$.

3. $\mathcal{T}_{\varepsilon}\left(\nabla u^{\varepsilon}\right) \rightarrow \nabla u_{0}+\nabla_{y} u_{1} \quad$ weakly in $\left[L^{2}(\Omega \times Y)\right]^{2}$.

4. The functions $u_{0}$ and $u_{1}$ satisfy the following unfolded limit problem:

$$
\begin{aligned}
& \frac{1}{|Y|} \int_{\Omega} \int_{Y} \sigma(y)\left(\nabla u_{0}(x)+\nabla_{y} u_{1}(x, y)\right) \cdot\left(\nabla v_{0}+\nabla_{y} v_{1}(x, y)\right) \mathrm{d} x \mathrm{~d} y \\
= & \left\langle f, v_{0}\right\rangle_{H^{-1}(\Omega), H_{0}^{1}(\Omega)}
\end{aligned}
$$

for all $v_{0} \in H_{0}^{1}(\Omega)$ and $v_{1} \in L^{2}\left(\Omega ; H_{\#}^{1}(Y)\right)$.

Proof. Let us recall that according to Theorem 2.8, the variational formulation (2.13) has a unique solution $u^{\varepsilon} \in H_{0}^{1}(\Omega)$ which satisfies in addition the uniform estimate

$$
\left\|\nabla u^{\varepsilon}\right\|_{L^{2}(\Omega)} \leqslant C
$$


Assertions 1, 2, 3 are easily obtained by following the steps of the proof of [16, Theorem 5.3].

In order to pass to the limit in the variational formulation (2.13) and obtain the last assertion, we choose particular test functions of the form

$$
v^{\varepsilon}(x)=\varphi_{0}(x)+\varepsilon \varphi_{1}(x) \psi\left(\frac{x}{\varepsilon}\right) \in H_{0}^{1}(\Omega),
$$

where $\varphi_{0}, \varphi_{1} \in \mathcal{D}(\Omega)$ and $\psi \in H_{\#}^{1}(Y)$. For the left-hand side we have by using $(3.1)$ and property $\left(\mathbf{P}_{2}\right)$ :

$$
\begin{aligned}
& \int_{\Omega} \sigma^{\varepsilon}(x) \nabla u^{\varepsilon}(x) \cdot \nabla v^{\varepsilon}(x) d x \sim \frac{1}{|Y|} \int_{\Omega} \int_{Y} \mathcal{T}_{\varepsilon}\left(\sigma^{\varepsilon} \nabla u^{\varepsilon} \cdot \nabla v^{\varepsilon}\right)(x, y) \mathrm{d} x \mathrm{~d} y \\
= & \frac{1}{|Y|} \int_{\Omega} \int_{Y} \mathcal{T}_{\varepsilon}\left(\sigma^{\varepsilon}\right)(x, y) \mathcal{T}_{\varepsilon}\left(\nabla u^{\varepsilon}\right)(x, y) \cdot \mathcal{T}_{\varepsilon}\left(\nabla v^{\varepsilon}\right)(x, y) \mathrm{d} x \mathrm{~d} y \\
= & \frac{1}{|Y|} \int_{\Omega} \int_{Y} \mathcal{T}_{\varepsilon}\left(\sigma^{\varepsilon}\right)(x, y) \mathcal{T}_{\varepsilon}\left(\nabla u^{\varepsilon}\right)(x, y) \cdot \mathcal{T}_{\varepsilon}\left(\nabla_{x} \varphi_{0}+\varepsilon \nabla_{x} \varphi_{1} \psi+\varphi_{1} \nabla_{y} \psi\right)(x, y) \mathrm{d} x \mathrm{~d} y .
\end{aligned}
$$

We pass to the limit $\varepsilon \rightarrow 0$ and by using the fact that $\mathcal{T}_{\varepsilon}\left(\sigma^{\varepsilon}\right)$ strongly converges to $\sigma(y)$ in $\left[L^{1}(\Omega \times Y)\right]^{2 \times 2}$ (recall that $\sigma^{\varepsilon}$ is uniformly bounded in $L^{\infty}\left(Y, \mathbb{R}^{2 \times 2}\right)$ ), assertion 3 above, and properties $\left(\mathbf{P}_{1}\right)-\left(\mathbf{P}_{4}\right)$, we obtain that:

$$
\begin{aligned}
& \lim _{\varepsilon \rightarrow 0} \int_{\Omega} \sigma^{\varepsilon}(x) \nabla u^{\varepsilon}(x) \cdot \nabla v^{\varepsilon}(x) \mathrm{d} x \\
= & \frac{1}{|Y|} \int_{\Omega} \int_{Y} \sigma(y)\left(\nabla u_{0}(x)+\nabla_{y} u_{1}(x, y)\right) \cdot\left(\nabla \varphi_{0}(x)+\varphi_{1}(x) \nabla_{y} \psi(x, y)\right) \mathrm{d} x \mathrm{~d} y .
\end{aligned}
$$

Similarly, for the right-hand side we have:

$$
\lim _{\varepsilon \rightarrow 0}\left\langle f, v^{\varepsilon}\right\rangle_{H^{-1}(\Omega), H_{0}^{1}(\Omega)}=\lim _{\varepsilon \rightarrow 0}\left\langle f, \varphi_{0}+\varepsilon \varphi_{1} \psi\left(\frac{\dot{\varepsilon}}{\varepsilon}\right)\right\rangle_{H^{-1}(\Omega), H_{0}^{1}(\Omega)}=\left\langle f, \varphi_{0}\right\rangle_{H^{-1}(\Omega), H_{0}^{1}(\Omega)}
$$

since $\varepsilon \varphi_{1} \psi\left(\frac{\cdot}{\varepsilon}\right)$ tends weakly to 0 in $H_{0}^{1}(\Omega)$.

Passing to the limit in $(2.13)$ we get for all $\varphi_{0}, \varphi_{1} \in \mathcal{D}(\Omega)$ and $\psi \in H_{\#}^{1}(Y)$ :

$$
\begin{aligned}
& \frac{1}{|Y|} \int_{\Omega} \int_{Y} \sigma(y)\left(\nabla u_{0}(x)+\nabla_{y} u_{1}(x, y)\right) \cdot\left(\nabla \varphi_{0}(x)+\nabla_{y}\left(\varphi_{1}(x) \psi(y)\right)\right) \mathrm{d} x \mathrm{~d} y \\
= & \left\langle f, \varphi_{0}\right\rangle_{H^{-1}(\Omega), H_{0}^{1}(\Omega)},
\end{aligned}
$$

and by the density of $\mathcal{D}(\Omega)$ in $H_{0}^{1}(\Omega)$ and $\mathcal{D}(\Omega) \otimes H_{\#}^{1}(Y)$ in $L^{2}\left(\Omega ; H_{\#}^{1}(Y)\right)$ we obtain that $\left(u_{0}, u_{1}\right)$ satisfies for all $v_{0} \in H_{0}^{1}(\Omega)$ and all $v_{1} \in L^{2}\left(\Omega ; H_{\#}^{1}(Y)\right)$ :

$$
\frac{1}{|Y|} \int_{\Omega} \int_{Y} \sigma(y)\left(\nabla u_{0}(x)+\nabla_{y} u_{1}(x, y)\right) \cdot\left(\nabla v_{0}(x)+\nabla_{y} v_{1}(x, y)\right) \mathrm{d} x \mathrm{~d} y=\left\langle f, v_{0}\right\rangle_{H^{-1}(\Omega), H_{0}^{1}(\Omega)} .
$$

3.2. Well-posedness of the unfolded limit problem. In this section, we construct an appropriate two scales $\mathbf{T}$-coercivity operator to show the well-posedness of the unfolded limit problem (3.2) under the contrast condition (1.3). 
Let $W^{\#}$ be the functional space defined by $(2.7)$ and let $\mathcal{H}=H_{0}^{1}(\Omega) \times L^{2}\left(\Omega ; W^{\#}\right)$. The Hilbert space $\mathcal{H}$ is endowed with the norm

$$
\|\mathcal{U}\|_{\mathcal{H}}:=\left\{\left\|\nabla u_{0}\right\|_{L^{2}(\Omega)}^{2}+\left\|\nabla_{y} u_{1}\right\|_{L^{2}(\Omega \times Y)}^{2}\right\}^{1 / 2}, \quad \forall \mathcal{U}=\left(u_{0}, u_{1}\right) \in \mathcal{H} .
$$

Let us emphasize that due to the periodicity of $u_{1}(x, \cdot)$, it follows from Green's formula that

$$
\int_{\Omega} \int_{Y} \nabla u_{0}(x) \cdot \nabla_{y} u_{1}(x, y) \mathrm{d} x \mathrm{~d} y=\int_{\Omega} \int_{Y} \nabla_{y} \cdot\left(\nabla u_{0}(x) u_{1}(x, y)\right) \mathrm{d} x \mathrm{~d} y=0,
$$

for all $U=\left(u_{0}, u_{1}\right) \in \mathcal{H}$. As a consequence, we have

$$
\left\|\nabla u_{0}(x)+\nabla_{y} u_{1}(x, y)\right\|_{L^{2}(\Omega \times Y)}^{2}=\|\mathcal{U}\|_{\mathcal{H}}^{2} .
$$

We define on $\mathcal{H}$ the bilinear form

$$
\mathcal{A}(\mathcal{U}, \mathcal{V}):=\frac{1}{|Y|} \int_{\Omega} \int_{Y} \sigma(y)\left(\nabla u_{0}(x)+\nabla_{y} u_{1}(x, y)\right) \cdot\left(\nabla v_{0}(x)+\nabla_{y} v_{1}(x, y)\right) \mathrm{d} x \mathrm{~d} y
$$

for all $\mathcal{U}=\left(u_{0}, u_{1}\right) \in \mathcal{H}$ and all $\mathcal{V}=\left(v_{0}, v_{1}\right) \in \mathcal{H}$. Given $\mathcal{F} \in \mathcal{H}^{\prime}\left(\mathcal{H}^{\prime}\right.$ denotes here the dual space of $\mathcal{H})$, consider following the variational problem:

Find $\mathcal{U}=\left(u_{0}, u_{1}\right) \in \mathcal{H}$ such that

$$
\mathcal{A}(\mathcal{U}, \mathcal{V})=\langle\mathcal{F}, \mathcal{V}\rangle_{\mathcal{H}^{\prime}, \mathcal{H}} \quad \forall \mathcal{V}=\left(v_{0}, v_{1}\right) \in \mathcal{H}
$$

Note that the limit problem (3.2) fits into the above variational framework provided we set $\langle\mathcal{F}, \mathcal{V}\rangle_{\mathcal{H}^{\prime}, \mathcal{H}}:=\left\langle f, v_{0}\right\rangle_{H^{-1}(\Omega), H_{0}^{1}(\Omega)}$ which clearly defines an element $\mathcal{F}$ of $\mathcal{H}^{\prime}$.

TheOREM 3.2. Let $\mathcal{F} \in \mathcal{H}^{\prime}$ and let $\kappa_{Y}$ denote the constant in (2.5). If the contrast $\kappa:=\left|\frac{\sigma_{e}}{\sigma_{i}}\right|$ satisfies

$$
\kappa>\kappa_{Y}
$$

then the variational problem (3.4) admits a unique solution $\mathcal{U}=\left(u_{0}, u_{1}\right) \in \mathcal{H}$ and there exists a constant $C>0$ such that

$$
\|\mathcal{U}\|_{\mathcal{H}} \leqslant C\|\mathcal{F}\|_{\mathcal{H}^{\prime}}
$$

Proof. Without loss of generality, we assume that $\sigma_{e}>0$ and $\sigma_{i}<0$ so that $\kappa=\sigma_{e} /\left|\sigma_{i}\right|$. Let $\mathbf{T}_{Y} \in \mathcal{L}\left(H^{1}(Y)\right)$ be the operator defined by (2.11). We note that $\mathbf{T}_{Y}$ defines an isomorphism on $H^{1}(Y)$ (one can check that $\left(\mathbf{T}_{Y}\right)^{2}=\mathrm{Id}$ ). Furthermore, since $P$ leaves invariant affine functions (according to assertion 1 of Lemma 2.2), we clearly have

$$
\mathbf{T}_{Y}(\xi \cdot y)=\xi \cdot y, \quad \forall \xi \in \mathbb{R}^{2}
$$

Now, we can define on $\mathcal{H}$ the operator

$$
\mathbf{T} \mathcal{U}=\left(u_{0}, \mathbf{T}_{Y} u_{1}\right), \quad \forall \mathcal{U}=\left(u_{0}, u_{1}\right) \in \mathcal{H}
$$


where for the sake of simplicity and by a slight abuse of notation, we have denoted by $\mathbf{T}_{Y} u_{1}$ the function defined on $\Omega \times Y$ by $\left(\mathbf{T}_{Y} u_{1}\right)(x, y)=\left(\mathbf{T}_{Y}\left(u_{1}(x, \cdot)\right)(y)\right.$. One can easily check that $\mathbf{T} \in \mathcal{L}(\mathcal{H})$ and defines an isomorphism on $\mathcal{H}\left(\right.$ with $\left.\mathbf{T}^{2}=\mathrm{Id}\right)$.

For every $\mathcal{U}=\left(u_{0}, u_{1}\right) \in \mathcal{H}$, we note that

$$
\nabla u_{0}(x)+\nabla_{y} u_{1}(x, y)=\nabla_{y}\left(\nabla u_{0}(x) \cdot y+u_{1}(x, y)\right) .
$$

Therefore, we have

$$
\mathcal{A}(\mathcal{U}, \mathbf{T} \mathcal{U})=\frac{1}{|Y|} \int_{\Omega} \int_{Y} \sigma(y) \nabla_{y}\left(\nabla u_{0}(x) \cdot y+u_{1}(x, y)\right) \cdot \nabla_{y}\left(\nabla u_{0}(x) \cdot y+\mathbf{T}_{Y} u_{1}(x, y)\right) \mathrm{d} x \mathrm{~d} y
$$

and by using (3.6)

$$
\mathcal{A}(\mathcal{U}, \mathbf{T} \mathcal{U})=\frac{1}{|Y|} \int_{\Omega} \int_{Y} \sigma(y) \nabla_{y}\left(\nabla u_{0}(x) \cdot y+u_{1}(x, y)\right) \cdot \nabla_{y} \mathbf{T}_{Y}\left(\nabla u_{0}(x) \cdot y+u_{1}(x, y)\right) \mathrm{d} x \mathrm{~d} y .
$$

Defining the function $y \mapsto U_{x}(y):=\nabla u_{0}(x) \cdot y+u_{1}(x, y)$ (which satisfies $U_{x} \in H^{1}(Y)$ for almost every $x \in \Omega$ ), we have

$$
\mathcal{A}(\mathcal{U}, \mathbf{T} \mathcal{U})=\frac{1}{|Y|} \int_{\Omega} \int_{Y} \sigma(y) \nabla_{y} U_{x}(y) \cdot \nabla_{y}\left(\mathbf{T}_{Y} U_{x}\right)(y) \mathrm{d} x \mathrm{~d} y=\frac{1}{|Y|} \int_{\Omega} a\left(U_{x}, \mathbf{T}_{Y} U_{x}\right) \mathrm{d} x
$$

where $a(\cdot, \cdot)$ is defined in $(2.9)$.

Following the proof of Theorem 2.5, one can easily show that there exists $\gamma>0$ such that

$$
a\left(U_{x}, \mathbf{T}_{Y} U_{x}\right) \geqslant \gamma\left\|\nabla_{y} U_{x}\right\|_{L^{2}(Y)}^{2}
$$

Integrating the above relation on $\Omega$ we immediately get that

$$
\mathcal{A}(\mathcal{U}, \mathbf{T} \mathcal{U}) \geqslant \gamma \int_{\Omega}\left\|\nabla_{y} U_{x}\right\|_{L^{2}(Y)}^{2} \mathrm{~d} x=\gamma\left\|\nabla u_{0}(x)+\nabla_{y} u_{1}(x, y)\right\|_{L^{2}(\Omega \times Y)}^{2}=\gamma\|\mathcal{U}\|_{\mathcal{H}}^{2},
$$

where the last equality follows from (3.3). We conclude by using Theorem 2.1.

\section{Analysis of the homogenized problem}

In the previous section, we have seen that $u^{\varepsilon}$ converges (in the sense of Theorem 3.1) to a limit $\left(u_{0}, u_{1}\right)$ solution of the unfolded limit problem (3.2). Let us emphasize that this problem reads as the classical unfolded limit problem obtained in the elliptic case (i.e. for coefficients $\sigma_{i}$ and $\sigma_{e}$ having the same sign). In view of numerical computations, a more convenient way to characterize the limit $\left(u_{0}, u_{1}\right)$ can be obtained by introducing the classical cell problems, which read in variational form as follows for $j=1,2$ (see (2.7) for the definition of the functional space $\left.W^{\#}\right)$ :

$$
\begin{gathered}
\text { Find } \chi_{j} \in W^{\#} \text { such that for all } v \in W^{\#}: \\
\int_{Y} \sigma(y) \nabla \chi_{j}(y) \cdot \nabla v(y) \mathrm{d} y=\int_{Y}\left(\sigma_{e} \mathbb{1}_{Y_{e}}(y)+\sigma_{i} \mathbb{1}_{Y_{i}}(y)\right) \frac{\partial v}{\partial y_{j}}(y) \mathrm{d} y .
\end{gathered}
$$

These two problems are non elliptic but they fit into the framework of $\S .2 .3$ (see (2.10)). Hence, according to Theorem 2.5, their well-posedness is ensured for

$$
\kappa>\kappa_{Y}
$$


where $\kappa_{Y}$ denote the constant in (2.6).

By following the same steps as in the elliptic case, one can now decouple the unfolded limit problem (3.2) to obtain a single boundary value problem equation for $u_{0}$ and express $u_{1}$ via $u_{0}, \chi_{1}, \chi_{2}$. More precisely, we can get the following result (see for instance [17, p. 184-185]).

Proposition 4.1. Assume that the contrast $\kappa:=\left|\frac{\sigma_{e}}{\sigma_{i}}\right|$ satisfies

$$
\kappa>\kappa_{Y}
$$

Let $\chi_{1}, \chi_{2}$ be the solutions of (4.1), and $\sigma^{H}=\left(\sigma_{j k}^{H}\right)_{1 \leqslant j, k \leqslant 2}$ be the $2 \times 2$ symmetric matrix defined by

$$
\sigma_{j k}^{H}=\frac{1}{|Y|} \int_{Y} \sigma(y) \nabla\left(y_{j}-\chi_{j}(y)\right) \cdot \nabla\left(y_{k}-\chi_{k}(y)\right) \mathrm{d} y .
$$

Then, the following assertions hold true:

1. If $\left(u_{0}, u_{1}\right) \in \mathcal{H}$ solve the unfolded limit problem (3.2), then $u_{0}$ solves the homogenized problem

$$
\begin{cases}-\operatorname{div}\left(\sigma^{H} \nabla u_{0}\right)=|Y| f, & \text { in } \Omega \\ u_{0}=0 & \text { on } \partial \Omega\end{cases}
$$

and $u_{1}$ is given by

$$
u_{1}(x, y)=-\sum_{j=1}^{2} \chi_{j}(y) \frac{\partial u_{0}}{\partial x_{j}}(x),
$$

2. Conversely, let $u_{0} \in H_{0}^{1}(\Omega)$ solve (4.3) and let $u_{1} \in L^{2}\left(\Omega ; W^{\#}\right)$ be defined by (4.4). Then $u:=\left(u_{0}, u_{1}\right) \in \mathcal{H}$ solves the unfolded limit problem (3.2).

The above result provides two equivalent formulations of the limit problem satisfied by $\left(u_{0}, u_{1}\right)$, a coupled and decoupled one. Due to the well-posedness result obtained in Theorem 3.2 for large enough contrasts, it also provides a well-posedness result for the homogenized problem (4.3), which is far from being obvious a priori.

It is worth noticing that the expression of the homogenized coefficients (4.2) is exactly the same as in the standard elliptic case, for which the homogenized tensor $\sigma^{H}$ is known to be symmetric definite positive (see for instance [17, p.115]). Indeed, according to formula (6.44) in [17], the following identity holds true for all $\xi=\left(\xi_{1}, \xi_{2}\right)^{T} \in \mathbb{R}^{2}$ :

$$
\sigma^{H} \xi \cdot \xi=\int_{\Omega} \sigma(y) \nabla u_{\xi}(y) \cdot \nabla u_{\xi}(y) \mathrm{d} y
$$

where

$$
u_{\xi}(y)=\xi_{1}\left(y_{1}-\chi_{1}(y)\right)+\xi_{2}\left(y_{2}-\chi_{2}(y)\right) .
$$

Equation (4.5) shows that $\sigma^{H}$ is positive in the elliptic case, but suggests that $\sigma^{H}$ can be indefinite in the case of sign changing coefficients. One needs to study the sign of the determinant of the diagonalizable matrix $\sigma^{H}$, which strongly depends on the inclusion $Y_{i}$ (shape and volume) and on the values of $\sigma_{i}$ and $\sigma_{e}$. Taking advantage of 
the isotropy of $\sigma$, let us derive more explicit expressions for the homogenized coefficients. Noting that the integrand appearing in the right-hand side of (4.1) can also be written $\sigma(y) \nabla y_{j} \cdot \nabla v(y)$, we obtain that the problem satisfied by $\chi_{j}, j=1,2$, is equivalent to

$$
\int_{Y} \sigma(y)\left(\nabla y_{j}-\nabla \chi_{j}(y)\right) \cdot \nabla v(y) \mathrm{d} y=0, \quad \forall v \in W^{\#} .
$$

In particular, we have

$$
\int_{Y} \sigma(y)\left(\nabla y_{j}-\nabla \chi_{j}(y)\right) \cdot \nabla \chi_{k}(y) \mathrm{d} y=0, \quad j, k=1,2 .
$$

Using this identity in the definition (4.2) of $\sigma^{H}$, we obtain that:

$$
\sigma^{H}=\left(\begin{array}{cc}
\bar{\sigma}-\rho_{1} & \tau \\
\tau & \bar{\sigma}-\rho_{2}
\end{array}\right)
$$

in which (below, $j=1,2$ ):

$$
\left\{\begin{array}{l}
\rho_{j}=-\frac{1}{|Y|} \int_{Y}\left(\sigma_{e} \mathbb{1}_{Y_{e}}(y)+\sigma_{i} \mathbb{1}_{Y_{i}}(y)\right) \frac{\partial \chi_{j}}{\partial y_{j}}(y) \mathrm{d} y=-\frac{1}{|Y|} \int_{Y} \sigma(y) \nabla \chi_{j}(y) \cdot \nabla \chi_{j}(y) \mathrm{d} y \\
\tau=-\frac{1}{|Y|} \int_{Y}\left(\sigma_{e} \mathbb{1}_{Y_{e}}(y)+\sigma_{i} \mathbb{1}_{Y_{i}}(y)\right) \frac{\partial \chi_{1}}{\partial y_{2}}(y) \mathrm{d} y=-\frac{1}{|Y|} \int_{Y} \sigma(y) \nabla \chi_{1}(y) \cdot \nabla \chi_{2}(y) \mathrm{d} y \\
\bar{\sigma}:=\frac{1}{|Y|} \int_{Y}\left(\sigma_{e} \mathbb{1}_{Y_{e}}(y)+\sigma_{i} \mathbb{1}_{Y_{i}}(y)\right) \mathrm{d} y=\alpha \sigma_{e}+(1-\alpha) \sigma_{i}
\end{array}\right.
$$

and

$$
\alpha:=\frac{\left|Y_{e}\right|}{|Y|}
$$
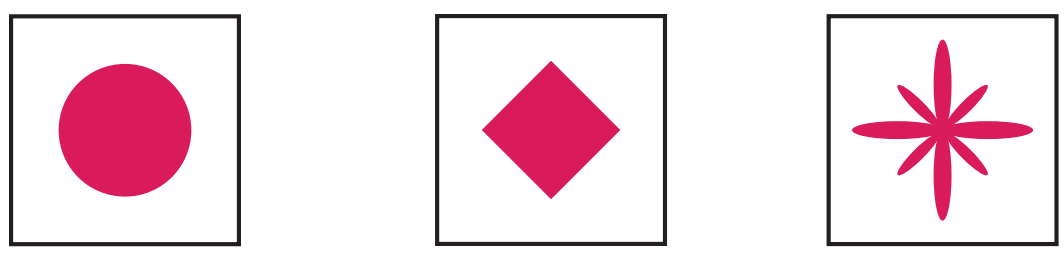

FIG. 4.1. Simple rotationally invariant geometries.

Proving theoretical results on the influence of the different parameters of the inclusion (shape and volume of the inclusions, average material conductivity $\bar{\sigma}$ ) on the definiteness of $\sigma^{H}$ seems to be a hard problem. Indeed, obtaining bounds for the coefficients of the homogenized matrix using Hashin-Shtrikman variational principle (see Allaire [1, p. 107]) is not trivial as it uses in a crucial way the ellipticity of $\sigma(y)$. Direct computations in two dimensions are possible for some specific configurations as detailed in Jikov, Kozlov, and Oleĭnik [22, p. 35]. However, one can check that the symmetry condition required there does not cover our main assumptions $\left(\partial Y_{i} \cap \partial Y=\varnothing\right.$ and $\left.\sigma_{i} \sigma_{e}<0\right)$. Consequently, a numerical study could be helpful to enlighten whether $\sigma^{H}$ is positive/negative or indefinite (see, for instance, Rohan, et al. [4, 28, 29] for such numerical investigations in phononics). As the numerical approximation of sign changing 
coefficients problems requires specific tools [14,15,25], this task is beyond the scope of this paper and will be the investigated in a forthcoming work.

However, more can be said about $\sigma^{H}$ in the particular case - but rather significant for applications - where $\sigma(y)$ is invariant by the rotation $\Theta_{\pi / 2}$ of angle $\pi / 2$ centered in the middle of the reference cell $Y$ (see Figure 4.1 for simple illustrations).

In particular, the next result shows that for such geometries, the obtained homogenized problem is always isotropic and definite (positive or negative) or degenerate.

Proposition 4.2. Assume that the reference cell is invariant under a rotation of angle $\Theta_{\pi / 2}$. Then, the coefficients of the homogenized tensor $\sigma^{H}$ defined by (4.7) satisfy

$$
\rho_{1}=\rho_{2}, \quad \tau=0 .
$$

Proof. Recall that if $\mathcal{O}=\Phi\left(\mathcal{O}^{\star}\right)$ is a given bounded Lipschitz domain obtained from a reference domain $\mathcal{O}^{\star}$ via a diffeomorphism $\Phi \in \mathscr{C}^{1}\left(\mathcal{O}^{\star}, \mathcal{O}\right)$, then

$$
\int_{\mathcal{O}} \nabla u(y) \cdot \nabla v(y) \mathrm{d} y=\int_{\mathcal{O}^{\star}} \nabla u^{\star}\left(y^{\star}\right) \cdot \mathbf{A} \nabla v^{\star}\left(y^{\star}\right) \mathrm{d} y^{\star},
$$

with (here, $D \Phi$ denotes the Jacobian of $\Phi$ )

$$
\begin{gathered}
u^{\star}\left(y^{\star}\right)=u(y)=(u \circ \Phi)\left(y^{\star}\right), \quad v^{\star}\left(y^{\star}\right)=v(y)=(v \circ \Phi)\left(y^{\star}\right) \\
\mathbf{A}=(D \Phi)^{-1}(D \Phi)^{-\mathbf{T}}|\operatorname{det} D \Phi| .
\end{gathered}
$$

According to (4.6), $\chi_{1}$ is characterized by:

$$
\int_{Y} \sigma(y)\left(\nabla y_{1}-\nabla \chi_{1}(y)\right) \cdot \nabla v(y) \mathrm{d} y=0, \quad \forall v \in W^{\#} .
$$

The change of variable $y^{\star}=\Theta_{\pi / 2} y=\left(-y_{2}, y_{1}\right)$ in the above relation yields immediately (using the fact that $\sigma(y)$ is by assumption invariant under a rotation of angle $\Theta_{\pi / 2}$ )

$$
\int_{Y} \sigma\left(y^{\star}\right)\left(\nabla y_{2}^{\star}-\nabla \chi_{1}^{\star}\left(y^{\star}\right)\right) \cdot \nabla v^{\star}\left(y^{\star}\right) \mathrm{d} y^{\star}=0, \quad \forall v^{\star} \in W^{\#},
$$

where we have set $\chi_{1}^{\star}\left(y^{\star}\right):=\chi_{1}(y)=\chi_{1}\left(\Theta_{-\pi / 2} y^{\star}\right)$. This shows that

$$
\chi_{2}\left(y^{\star}\right)=\chi_{1}\left(\Theta_{-\pi / 2} y^{\star}\right), \quad \forall y^{\star} \in Y .
$$

This implies in particular that

$$
\begin{aligned}
\rho_{1} & =-\frac{1}{|Y|} \int_{Y} \sigma(y) \nabla \chi_{1}(y) \cdot \nabla \chi_{1}(y) \mathrm{d} y \\
& =-\frac{1}{|Y|} \int_{Y} \sigma\left(y^{\star}\right) \nabla \chi_{2}\left(y^{\star}\right) \cdot \nabla \chi_{2}\left(y^{\star}\right) \mathrm{d} y^{\star}=\rho_{2}=: \rho,
\end{aligned}
$$

and

$$
\tau=-\frac{1}{|Y|} \int_{Y} \sigma(y) \nabla y_{2} \cdot \nabla \chi_{1}(y) \mathrm{d} y=\frac{1}{|Y|} \int_{Y} \sigma\left(y^{\star}\right) \nabla y_{1}^{\star} \cdot \nabla \chi_{2}\left(y^{\star}\right) \mathrm{d} y^{\star}=-\tau .
$$

Hence, $\tau=0$. 
REMARK 4.3. The above result shows that setting $\rho$ the common value of $\rho_{1}$ and $\rho_{2}$, we have

$$
\sigma^{H}=(\bar{\sigma}-\rho)\left(\begin{array}{ll}
1 & 0 \\
0 & 1
\end{array}\right)
$$

and hence, $\sigma^{H}$ is positive (respectively negative) definite if and only if $\alpha, \sigma_{e}$ and $\sigma_{i}$ are chosen such that

$$
\rho<\bar{\sigma} \quad(\text { respectively } \rho>\bar{\sigma}) \text {. }
$$

\section{REFERENCES}

[1] G. Allaire, Shape Optimization by the Homogenization Method, Appl. Math. Sci., Springer-Verlag, New York, 146, 2002.

[2] G. Allaire, A brief introduction to homogenization and miscellaneous applications, in Mathematical and Numerical Approaches for Multiscale Problem, ESAIM Proc., EDP Sci., Les Ulis, 37, 1-49, 2012.

[3] H. Ammari, G. Ciraolo, H. Kang, H. Lee, and K. Yun, Spectral analysis of the Neumann-Poincaré operator and characterization of the stress concentration in anti-plane elasticity, Arch. Ration. Mech. Anal., 208, 275-304, 2013.

[4] A. Ávila, G. Griso, B. Miara, and E. Rohan, Multiscale modeling of elastic waves: theoretical justification and numerical simulation of band gaps, Multiscale Model. Simul., 7, 1-21, 2008.

[5] A.-S. Bonnet-Ben Dhia, L. Chesnel, and P.J. Ciarlet, T-coercivity for scalar interface problems between dielectrics and metamaterials, ESAIM Math. Model. Numer. Anal., 46, 1363-1387, 2012.

[6] A.-S. Bonnet-Ben Dhia, L. Chesnel, and P.J. Ciarlet, T-coercivity for the Maxwell problem with sign-changing coefficients, Comm. Part. Diff. Eqs., 39, 1007-1031, 2014.

[7] A.-S. Bonnet-Ben Dhia, P.J. Ciarlet, and C.M. Zwölf, Time harmonic wave diffraction problems in materials with sign-shifting coefficients, J. Comput. Appl. Math., 2008.

[8] A.-S. Bonnet-BenDhia, L. Chesnel, and H. Haddar, On the use of T-coercivity to study the interior transmission eigenvalue problem, C.R. Acad. Sci., Ser. I, 349, 647-651, 2011.

[9] G. Bouchitté and C. Bourel, Homogenization of finite metallic fibers and 3D-effective permittivity tensor, Tech. report, Université de Toulon, 2009.

[10] G. Bouchitté and C. Bourel, Multiscale nanorod metamaterials and realizable permittivity tensors, Commun. Comput. Phys., 11, 489-507, 2012.

[11] G. Bouchitté and D. Felbacq, Homogenization of a wire photonic crystal: the case of small volume fraction, SIAM J. Appl. Math., 66, 2061-2084 (electronic), 2006.

[12] G. Bouchitté and B. Schweizer, Cloaking of small objects by anomalous localized resonance, Quart. J. Mech. Appl. Math., 63, 437-463, 2010.

[13] F. Cakoni, D. Colton, and H. Haddar, The linear sampling method for anisotropic media, J. Comput. Appl. Math., 146, 285-299, 2002.

[14] L. Chesnel and P.J. Ciarlet, T-coercivity and continuous Galerkin methods: application to transmission problems with sign changing coefficients, Numer. Math., 124, 1-29, 2013.

[15] E.T. Chung and P.J. Ciarlet, A staggered discontinuous Galerkin method for wave propagation in media with dielectrics and meta-materials, J. Comput. Appl. Math., 239, 189-207, 2013.

[16] D. Cioranescu, A. Damlamian, and G. Griso, The periodic unfolding method in homogenization, SIAM J. Math. Anal., 40, 1585-1620, 2008.

[17] D. Cioranescu and P. Donato, An Introduction to Homogenization, Oxford Lecture Series in Mathematics and its Applications, The Clarendon Press, Oxford University Press, New York, $17,1999$.

[18] D. Cioranescu and J. Saint Jean Paulin, Homogenization of Reticulated Structures, Appl. Math. Sci., Springer-Verlag, New York, 136, 1999.

[19] D. Felbacq and G. Bouchitté, Homogenization of a set of parallel fibres, Waves in Random Media, 7, 245-256, 1997.

[20] P. Fernandes and M. Raffetto, Existence, uniqueness and finite element approximation of the solution of time-harmonic electromagnetic boundary value problems involving metamaterials, COMPEL, 24, 1450-1469, 2005.

[21] Z. Jacob, L.V. Alekseyev, and E. Narimanov, Optical hyperlens: Far-field imaging beyond the diffraction limit, Opt. Express, 14, 8247-8256, 2006. 


\section{HOMOGENIZATION OF MATERIALS WITH SIGN CHANGING COEFFICIENTS}

[22] V.V. Jikov, S.M. Kozlov, and O.A. Olĕnik, Homogenization of Differential Operators and Integral Functionals, Springer-Verlag, Berlin, 1994.

[23] J. Li and Y. Huang, Time-domain Finite Element Methods for Maxwell's Equations in Metamaterials, Springer Series in Comput. Math., Springer, Heidelberg, 43, 2013.

[24] G.W. Milton and N.A.P. Nicorovici, On the cloaking effects associated with anomalous localized resonance, Proc. R. Soc. Lond. Ser. A Math. Phys. Eng. Sci., 462, 3027-3059, 2006.

[25] S. Nicaise and J. Venel, A posteriori error estimates for a finite element approximation of transmission problems with sign changing coefficients, J. Comput. Appl. Math., 235, 4272-4282, 2011.

[26] G. Oliveri and M. Raffetto, A warning about metamaterials for users of frequency-domain numerical simulators, IEEE Trans. Antennas and Propagation, 56, 792-798, 2008.

[27] J.B. Pendry and S.A. Ramakrishna, Refining the perfect lens, Physica B: Condensed Matter, 338, 329-332, 2003.

[28] E. Rohan and B. Miara, Homogenization and shape sensitivity of microstructures for design of piezoelectric bio-materials, Mechanics of Advanced Materials and Structures, 13, 473-485, 2006.

[29] E. Rohan, B. Miara, and F. Seifrt, Numerical simulation of acoustic band gaps in homogenized elastic composites, Internat. J. Engrg. Sci., 47, 573-594, 2009.

[30] A. Salandrino and N. Engheta, Far-field subdiffraction optical microscopy using metamaterial crystals: Theory and simulations, Phys. Rev. B, 74, 075103, 2006.

[31] E. Shamonina and L. Solymar, Metamaterials: How the subject started, Metamaterials, 1, 12-18, 2007.

[32] D.R. Smith, J.B. Pendry, and M.C.K. Wiltshire, Metamaterials and negative refractive index, Science, 305, 788-792, 2004.

[33] S. Tricarico, F. Bilotti, and L. Vegni, Scattering cancellation by metamaterial cylindrical multilayers, Journal of the European Optical Society - Rapid publications, 4, 2009. 